\begin{tabular}{|c|l|}
\hline Title & Systematic study of the glass transition in poly hydric al cohols \\
\hline Author(s) & Nakanishi, Masahiro; Nozaki, Ryusuke \\
\hline Citation & $\begin{array}{l}\text { Physical Review E, 83(5), 051503 } \\
\text { https://doi.org/10.1103/PhysRevE.83.051503 }\end{array}$ \\
\hline Issue Date & 2011-05 \\
\hline Doc URL & http://hdl.handle.net/2115/45608 \\
\hline Rights & O2011 A merican Physical Society \\
\hline Type & article \\
\hline File Information & PRE83_5_051503.pdf \\
\hline
\end{tabular}

Instructions for use 


\title{
Systematic study of the glass transition in polyhydric alcohols
}

\author{
Masahiro Nakanishi (中西真大) ${ }^{*}$ and Ryusuke Nozaki (野峷龍介) \\ Department of Physics, Faculty of Science, Hokkaido University, Sapporo, Hokkaido 060-0810, Japan \\ (Received 25 November 2010; revised manuscript received 2 February 2011; published 13 May 2011)
}

\begin{abstract}
We have investigated the glass transitions of trihydric alcohols using broadband dielectric spectroscopy, and compare the results with those previously reported for sugar alcohols. Although a systematic glass transition feature related to molecular size has been reported for sugar alcohols, the essential factor governing this feature is still unclear because the number of carbon atoms $\left(N_{\mathrm{C}}\right)$ and the number of $\mathrm{OH}$ groups $\left(N_{\mathrm{OH}}\right)$ per molecule are identical in sugar alcohols. By examining trihydric alcohols $\left(N_{\mathrm{C}} \neq N_{\mathrm{OH}}\right)$, we conclude that $N_{\mathrm{OH}}$ is dominant for the characteristics of the slow dynamics, such as fragility and glass transition temperature. This result suggests that the topological structure of the hydrogen-bonding network (coordination number) plays an important role in the glass transition of polyhydric alcohols. Furthermore, the orientational correlation factor evaluated using the Kirkwood-Fröhlich theory reveals a similarity in hydrogen bond formation among a variety of polyhydric alcohols. Based on these two experimental results, we discuss a possible physical picture of the glass transition of polyhydric alcohols.
\end{abstract}

DOI: 10.1103/PhysRevE.83.051503

PACS number(s): 64.70.pm, 61.25.Em, 77.22.Gm

\section{INTRODUCTION}

The glass transition is currently one of the most discussed problems in condensed matter physics. Generally, in the supercooled state, which is the precursor to the glass transition, the structural relaxation time lengthens dramatically and eventually exceeds the time scale of experimental observation at the glass transition temperature $T_{g}$. Despite considerable experimental and theoretical effort, the mechanism of this "slowing down" is still unclear [1,2].

Since molecular dynamics are dominated by strong cooperativity in the supercooled state, the exact theoretical description from the first principle does not appear in the literature. Consequently, there is no microscopic understanding of the origin of the Vogel-Fulcher-Tammann (VFT) law, slow relaxation, dynamic crossovers, and other effects of glass transition. For this reason, an efficient approximation or coarse-grained model, which extracts the essential nature of the glass transition, needs to be found. Therefore, systematic experimental investigations are required to clarify the dominant parameter or pattern which produces the characteristic slowing down of the structural relaxation.

The temperature dependence of the relaxation time in the supercooled state is often characterized by fragility [3] and $T_{g}$, and the link between these quantities and chemical structure has been discussed for a long time [4-9]. In polymeric materials, Kunal et al. have found the qualitative relation between fragility and $T_{g}$ and stiffness of chain: Fragility is dominated by relative flexibility of the side group compared to flexibility of the backbone, whereas $T_{g}$ depends primarily on the backbone flexibility and the side group bulkiness [5]. For low-molecular weight molecules, on the other hand, León et al. have reported that the fragility and $T_{g}$ of the propylene glycol oligomer systematically increase with an increasing degree of polymerization [6]. Such a systematic change in fragility and $T_{g}$ is also known for smaller molecules, such as sugar alcohols. A sugar alcohol molecule is composed of a

*nakanishi@dielectrics.sci.hokudai.ac.jp linear carbon backbone chain and $\mathrm{OH}$ groups attached to each carbon atom (Fig. 1). It has been reported that sugar alcohols exhibit systematic increases in fragility and $T_{g}$ with increasing chain length [7-9].

When one discusses such a systematic trend, molecular weight (or an equivalent quantity, such as degree of polymerization and chain length) is most frequently chosen as the independent variable. However, molecular weight is often coupled with other quantities. For example, in the case of sugar alcohols the number of $\mathrm{OH}$ groups per molecule is proportional to the molecular weight, and in propylene glycol oligomers the number of ether bonds, the acceptors of hydrogen bonding, is also proportional to the molecular weight. Nevertheless, it has not been determined whether either of these quantities actually affects the glass transition behavior. Thus the dominant parameter determining the systematic trends in glass transition behavior remains unclear.

The dominant parameter is an essential piece of information for the construction of a coarse-grained model of the glass transition. Based on the experimental results for sugar alcohols mentioned previously, one can suggest two possible candidates for the dominant parameter; the number of carbon atoms in the main chain $\left(N_{\mathrm{C}}\right)$ and the number of $\mathrm{OH}$ groups $\left(N_{\mathrm{OH}}\right)$. The former can be related to the excluded volume as discussed in the free-volume model [10], whereas the latter is related to the number of connecting sites per molecule or, equivalently, to the topological structure of the hydrogen-bonding network [11].

In this paper, the glass transition behaviors for several polyhydric alcohols (Fig. 1) are compared systematically, and the parameter which dominates fragility and $T_{g}$ are discussed based on their molecular structure. As can be seen in Fig. 1, $N_{\mathrm{C}}=N_{\mathrm{OH}}$ for sugar alcohols, while for trihydric alcohols $N_{\mathrm{OH}}=3$ and $N_{\mathrm{C}} \neq N_{\mathrm{OH}}$ except for glycerol. Therefore, it is possible to determine which of $N_{\mathrm{C}}$ and $N_{\mathrm{OH}}$ is dominant for glass transition behavior. The trihydric alcohols with $N_{\mathrm{C}}=3$ through 7 are examined here [12]. Their properties are also compared with the previously reported results for sugar alcohols, DL-threitol, xylitol, and D-sorbitol [9]. The values of $N_{\mathrm{C}}$ and $N_{\mathrm{OH}}$ of these materials are listed in Table I. 


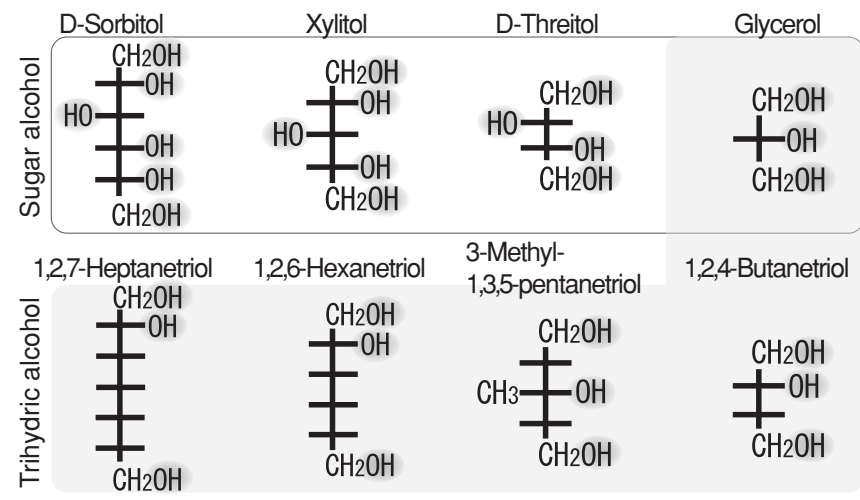

FIG. 1. Molecular structure of trihydric alcohols and sugar alcohols. Structures of sugar alcohols are shown as Fischer projections.

\section{EXPERIMENT}

Broadband dielectric spectroscopy (BDS) measurements covering 14 decades of frequency were carried out using three instruments: a self-made time-domain spectrometer $\left(10^{-6}\right.$ $\left.10^{-1} \mathrm{~Hz}\right)$, a self-made ac phase analyzer $\left(10^{-2}-10^{3} \mathrm{~Hz}\right)$, and an impedance analyzer (Agilent 4294A: $10^{2}-10^{8} \mathrm{~Hz}$ ). Details of the experimental setup have already been described elsewhere $[11,13]$. Our samples of trihydric alcohols were obtained from several sources. The 97\% 1,2,6-hexanetriol and the $98.5 \%$ glycerol were purchased from Merck KGaA and the Kishida Chemical Co., Ltd., respectively. The $97 \%$ 1,2,4-butanetriol, 80\% 3-methyl-1,3,5-pentanetriol (3MPT), and $95 \%$ 1,2,7-heptanetriol were purchased from the Tokyo Chemical Industry Co., Ltd.

For sorbitol and xylitol, density was measured using a pycnometer, and the refractive index was measured using a commercial refractometer (RX-5000 $\alpha$, ATAGO Co., Ltd.). These measurements were carried out in order to evaluate the Kirkwood-Fröhlich orientational correlation factor, because sorbitol and xylitol are solid at room temperature and no values in the supercooled state are available in the literature [14].

\section{RESULTS AND DISCUSSION}

The shapes of the dielectric spectra for the trihydric alcohols are very similar to each other. As a representative of them, spectra of heptanetriol at various temperatures are shown in

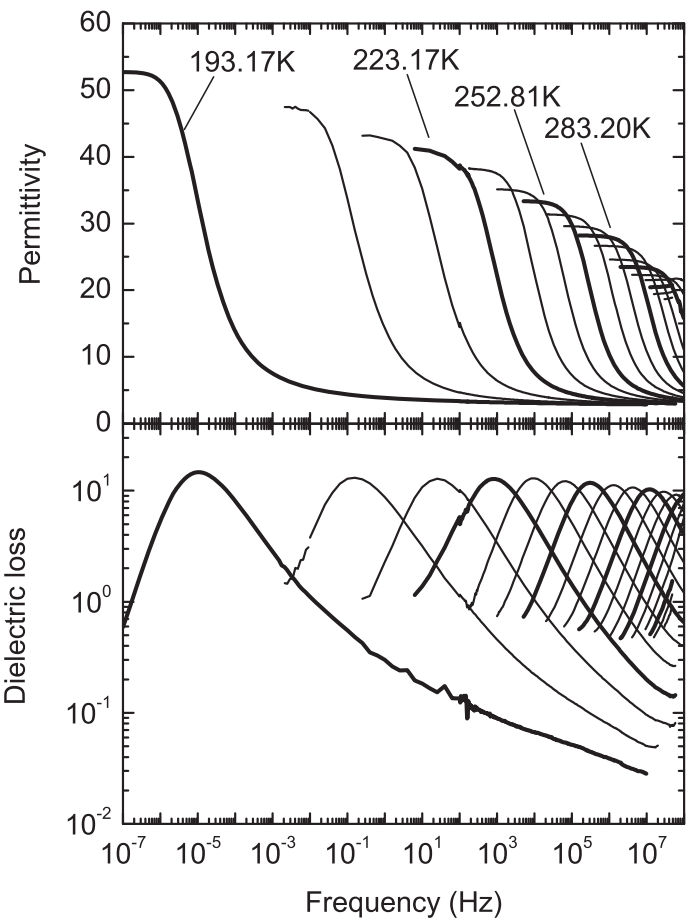

FIG. 2. Frequency dependence of complex permittivity (permittivity and dielectric loss) of 1,2,7-heptanetriol. Data at every $10 \mathrm{~K}$ from 193 to $363 \mathrm{~K}$ is shown.

Fig. 2. In each spectrum, a primary relaxation ( $\alpha$ relaxation) accompanied by an excess wing on its high-frequency flank is observed, as shown in hexanetriol [11] and glycerol [15]. These spectra can be reproduced by the superposition of a HavriliakNegami (HN) function [16] and a Cole-Cole function [17] for the $\alpha$ relaxation and excess wing, respectively. To discuss the spectral shape of the $\alpha$ relaxation, stretch exponents of the Kohlrausch-Williams-Watts (KWW) function $\left(\beta_{\mathrm{KWW}}\right)$ [18] are calculated from the parameters of the $\mathrm{HN}$ function using the transformation equation presented in Ref [19]. Thus calculated values of $\beta_{\mathrm{KWW}}$ at $T_{g}$ are listed in Table I. As can be recognized from these values, trihydric alcohols and sugar alcohols exhibit non-Debye features around $T_{g}$.

In Fig. 3, the peak frequencies of $\alpha$ relaxation of the trihydric alcohols are plotted against reciprocal temperature, together with the previously reported data for threitol, xylitol,

TABLE I. Fitting parameters for the VFT equation and several characteristic quantities for trihydric alcohols and sugar alcohols [9]. $\beta_{\mathrm{KWW}}\left(T_{g}\right)$ is KWW exponent at $T_{g} . \rho$ and RI stand for density and refractive index, respectively.

\begin{tabular}{lcccccccccc}
\hline \hline Sample & $N_{\mathrm{C}}$ & $N_{\mathrm{OH}}$ & $T_{0}(\mathrm{~K})$ & $\log _{10}\left[f_{0}(\mathrm{~Hz})\right]$ & $B(\mathrm{~K})$ & $T_{g}(\mathrm{~K})$ & $m$ & $\beta_{\mathrm{KWw}}\left(T_{g}\right)$ & $\rho\left(\mathrm{g} / \mathrm{cm}^{3}\right)$ & $\mathrm{RI}$ \\
\hline Sorbitol & 6 & 6 & 229.1 & 12.9 & 1350.3 & 268.3 & 102.3 & 0.404 & 1.44 \\
Xylitol & 5 & 5 & 197.8 & 13.8 & 1818.3 & 247.8 & 78.3 & 0.469 & 1.38 & 1.53 \\
Threitol & 4 & 4 & 175.0 & 13.5 & 1828.4 & 226.3 & 68.4 & 0.518 & $\mathrm{n} / \mathrm{a}$ & $\mathrm{n} / \mathrm{a}$ \\
Glycerol & 3 & 3 & 129.1 & 14.3 & 2349.7 & 191.7 & 50.0 & 0.633 & $1.2613^{\mathrm{a}}$ & $1.4746^{\mathrm{a}}$ \\
Butanetriol & 4 & 3 & 152.5 & 12.6 & 1614.3 & 200.7 & 60.7 & 0.626 & $1.18^{\mathrm{a}}$ & $1.4688^{\mathrm{a}}$ \\
3MPT & 5 & 3 & 152.3 & 12.8 & 1820.7 & 205.7 & 57.0 & 0.585 & $1.106^{\mathrm{b}}$ & $1.477^{\mathrm{b}}$ \\
Hexanetriol & 6 & 3 & 160.6 & 11.8 & 1309.3 & 201.9 & 67.6 & 0.564 & $1.1049^{\mathrm{a}}$ & $1.58^{\mathrm{a}}$ \\
Heptanetriol & 7 & 3 & 161.3 & 11.3 & 1194.0 & 200.3 & 68.2 & 0.527 & $\mathrm{n} / \mathrm{a}$ & $\mathrm{n} / \mathrm{a}$ \\
\hline \hline
\end{tabular}

${ }^{\mathrm{a}}$ Reference [30].

${ }^{\mathrm{b}}$ Reference [31]. 


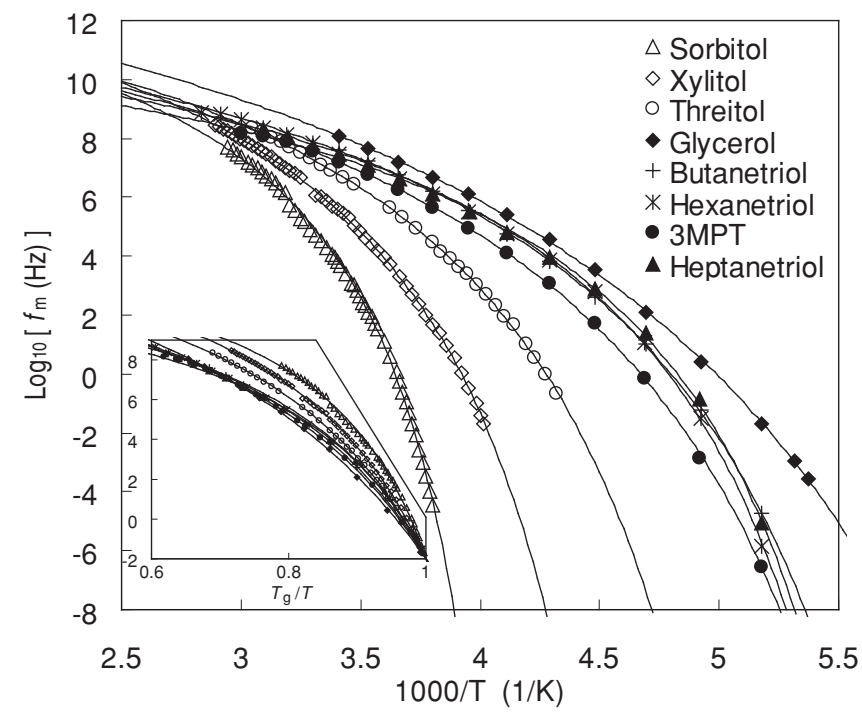

FIG. 3. Arrhenius diagram for trihydric alcohols and sugar alcohols. Peak frequency of $\alpha$ relaxation is plotted. Solid curves show best fit using the VFT equation. Data for sugar alcohols are taken from Ref. [9].

and sorbitol [9]. All the loci for the trihydric alcohols are close to each other, and their curvatures are smaller than those of sugar alcohols. These data can be reproduced by a VFT equation, $f_{\max }=f_{0} \exp \left[-B /\left(T-T_{0}\right)\right]$, where $f_{\max }$ is the peak frequency, $T$ is temperature, and $f_{0}, B$, and $T_{0}$ are fitting parameters. The results of least-squares fitting are shown as solid curves in Fig. 3, and the fitting parameters obtained are listed in Table I. Herein the glass transition temperature $T_{g}$ is defined as the temperature at which $f_{\max }=10^{-2} \mathrm{~Hz}$, and it can be calculated from the VFT parameters. The fragility $m$ can also be calculated from the VFT equation, according to $m=-d\left(\log _{10} f_{m}\right) /\left.d\left(T_{g} / T\right)\right|_{T=T_{g}}$. The values of $T_{g}$ and $m$ are also listed in Table I.

To compare the glass transition behavior between trihydric alcohols and sugar alcohols, $m, T_{g}$, and $T_{0}$ are plotted against $N_{\mathrm{C}}$ and $N_{\mathrm{OH}}$ in Fig. 4. As reported in previous works [7-9], all three parameters for sugar alcohols show a strong dependence on both $N_{\mathrm{C}}$ and $N_{\mathrm{OH}}$ (filled symbols). However, since $N_{\mathrm{C}}=$ $N_{\mathrm{OH}}$ for sugar alcohols, the figures are identical and it is impossible to separate the effects of the two parameters. For trihydric alcohols, on the other hand, $N_{\mathrm{OH}}$ is fixed at 3 , and $N_{\mathrm{C}}$ is gradually varied from 3 to 7 (open symbols). Thus distinct contributions from $N_{\mathrm{C}}$ become apparent. As can been seen in Fig. 4(b), $m$ shows only a weak $N_{\mathrm{C}}$ dependence compared to $N_{\mathrm{OH}}$. As a result, all $m$ of trihydric alcohols are gathered around $60 \pm 10$. Therefore, we conclude that $N_{\mathrm{OH}}$ is more dominant for fragility rather than $N_{\mathrm{C}}$. This can also be recognized as a systematic trend against $N_{\mathrm{OH}}$ in Fig. 4(a). The same trend is also found in $T_{g}$ and $T_{0}$, especially more clearly in $T_{g}$. Two characteristic temperatures, $T_{g}$ and $T_{0}$, are practically constant against $N_{\mathrm{C}}$ [Fig. 4(d)]. As a result, $T_{g}$ and $T_{0}$ systematically increase with increasing $N_{\mathrm{OH}}$ [Fig. 4(c)]. Thus, we again conclude that $N_{\mathrm{OH}}$ is more dominant for $T_{g}$ and $T_{0}$ than $N_{\mathrm{C}}$.

Generally, fragility and $T_{g}$ are discussed as characteristic values of the temperature dependence of structural relaxation time. From the present comparison between sugar alcohols and

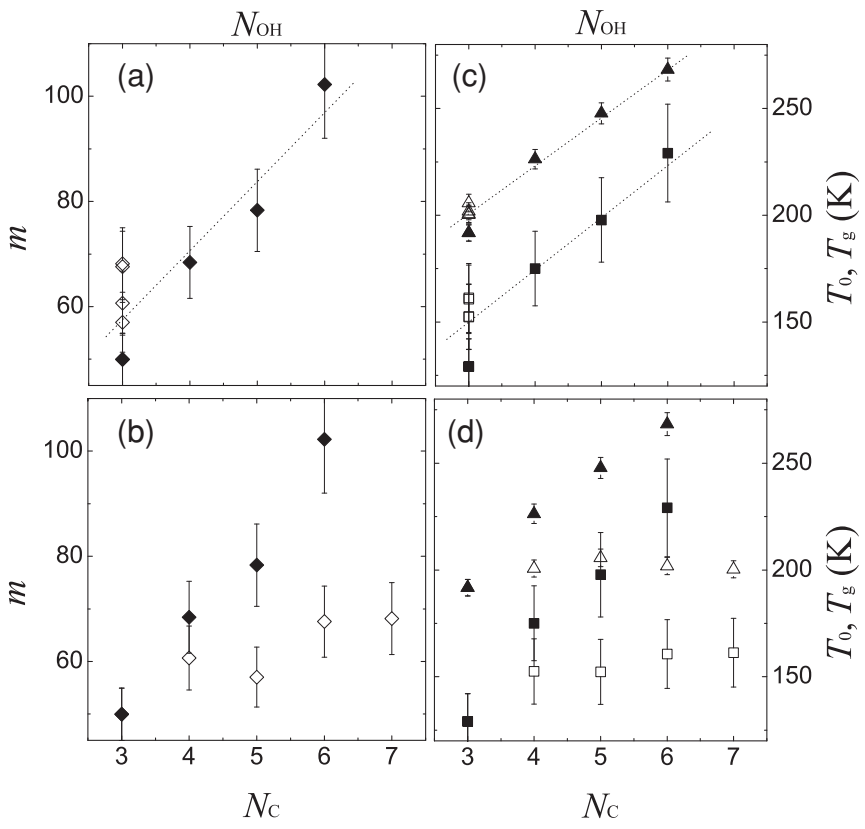

FIG. 4. Plots of fragility $(m), T_{g}$, and $T_{0}$ against $N_{C}$ and $N_{O H}$ for sugar alcohols (filled symbols) and trihydric alcohols (open symbols). Diamond, triangle and square show $m, T_{g}$, and $T_{0}$, respectively.

trihydric alcohols, it is strongly suggested that the glass transition behavior of polyhydric alcohols is mainly dominated by $N_{\mathrm{OH}}$ rather than by $N_{\mathrm{C}}$. Despite heptanetriol having more than twice the molecular weight of glycerol, the values of $m$ and $T_{g}$ for the two alcohols are very close to each other. Therefore, it can be concluded that the size of the molecules has a relatively small effect on the glass transition behavior of polyhydric alcohols. This is consistent with the experimental result that for propylene glycol oligomer at elevated pressure, the presence of hydrogen bonding reduces the volume effect [20].

In a hydrogen-bonding liquid, the carbon backbone chain and its $\mathrm{OH}$ groups form a hydrogen-bonding network. Chelli et al. have performed a molecular dynamics (MD) simulation for glycerol. They reported that there are large clusters of glycerol molecules connected through hydrogen bonds, and that the lifetime of these clusters is strongly correlated with diffusion time [21]. On the basis of this network picture, our experimental result suggests that the topology of the hydrogen-bonding network (coordination number) plays a key role in the glass transition of a hydrogen-bonding liquid. In the hydrogen-bonding network, polyhydric alcohol molecules can be regarded as nodes of the network, and $\mathrm{OH}$ groups attached to the backbone chain form links to neighboring molecules, according to a certain probability.

The importance of the role of $N_{\mathrm{OH}}$ is supported by two previously reported experimental results. First, fragility and $T_{g}$ for an ethylene glycol-water mixture are approximately insensitive to the mixing rate [22]. This can be attributed to the similarity of topological network structures, because both ethylene glycol and water have $N_{\mathrm{OH}}=2$. Second, it is reported that mixing water into sugar alcohols, such as sorbitol $\left(N_{\mathrm{OH}}=\right.$ 6) and xylitol $\left(N_{\mathrm{OH}}=5\right)$, causes a significant plasticizer effect, where fragility and $T_{g}$ are decreased as the concentration of water is increased $[23,24]$. This can be attributed to the fact 


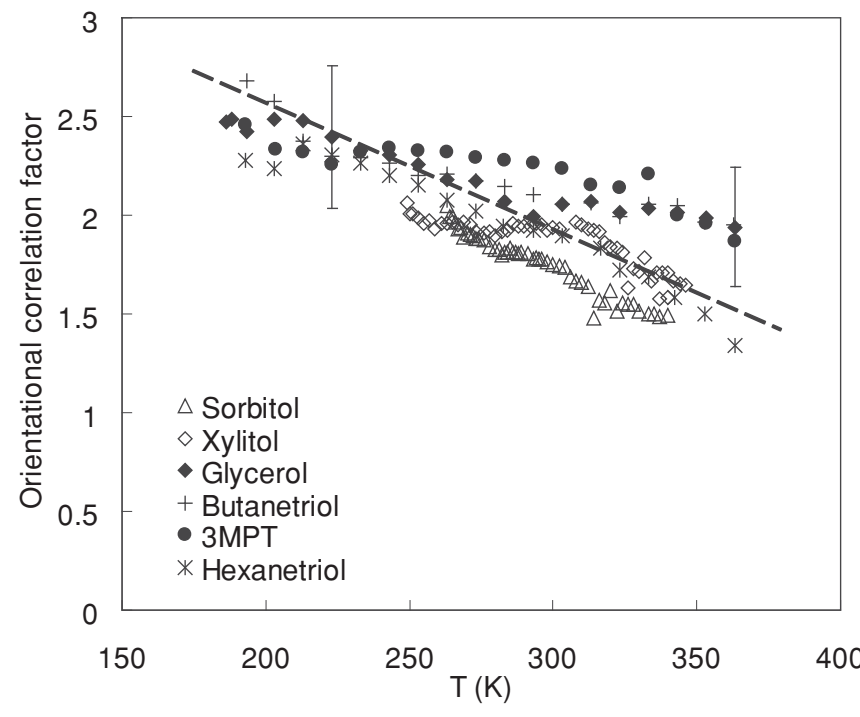

FIG. 5. Temperature dependence of the orientational correlation factor of $\mathrm{OH}$ groups for various polyhydric alcohols. The dashed line is drawn just to provide a common reference.

that the water (with $N_{\mathrm{OH}}=2$ ) decreases the mean coordination number of the network.

Such a network feature has been often discussed in covalent network glasses. For example, in the ternary system Ge-As-Se, the value of $T_{g}$ depends only on the coordination number and is independent of composition [25,26]. This implies the importance of topological structure. Although such composition dependence of coordination number is frequently discussed for covalent network glasses, the temperature dependence is rarely discussed. On the other hand, in hydrogen-bondingnetwork glasses, the probability for hydrogen-bonding formation depends significantly on temperature, as shown by MD simulation [21]. Therefore, the mean coordination number of a hydrogen-bonding molecule is also dependent on temperature, and cannot be fixed at any given value.

To evaluate the hydrogen-bond formation, the orientational correlation of $\mathrm{OH}$ groups is estimated using the Kirkwood-Fröhlich theory [27,28]: $\varepsilon_{s}-\varepsilon_{\infty}=$ $\left[3 \varepsilon_{s} /\left(2 \varepsilon_{s}+\varepsilon_{\infty}\right)\right]\left[\left(\varepsilon_{\infty}+2\right) / 3\right]^{2} n g \mu_{0}^{2} / 3 \varepsilon_{0} k_{B} T$, where $\mu_{0}$ is the isolated dipole moment, $\varepsilon_{s}$ is the static dielectric constant, $\varepsilon_{\infty}$ is the high-frequency limit of the dielectric constant, $n$ is the number density of dipole moments, $\varepsilon_{0}$ is the dielectric constant of the vacuum, and $k_{B}$ is the Boltzmann constant. The factor $g=(1+z\langle\cos \theta\rangle)$ is called the orientational correlation factor. This expresses the orientational correlation between a particular dipole moment and its $z$ neighbors. For $g>1$ dipoles prefer to lie parallel, while for $g<1$ they lie antiparallel. Usually, the dipole moment of the whole molecule is chosen as the unit dipole moment, $\mu_{0}$, and it is evaluated from the dielectric constant in the gas phase. However, for the polyhydric alcohols, the dipole moment of the whole molecule is not applicable because each $\mathrm{OH}$ group can rotate individually. For this reason, the dipole moment of an $\mathrm{OH}$ group (rather than the whole molecule) is chosen as $\mu_{0}$. Then, $g$ indicates the orientational correlation between $\mathrm{OH}$ groups. When two $\mathrm{OH}$ groups form a hydrogen bond, they have a value of $g$ within a certain range, indicating parallel orientation. Therefore, the orientational correlation factor $g$ with respect to the $\mathrm{OH}$ group reflects the formation of hydrogen bonds [11]. In this paper $\mu_{0}=1.69 \mathrm{D}$ is used for the dipole moment of an $\mathrm{OH}$ group [29]. The values of $\varepsilon_{\infty}$ are calculated from the square of the refractive index. The values of density and refractive index used are listed in Table I. It should be noted that a mismatch between $\varepsilon_{\infty}$ and the square of the refractive index is pointed out in Debye-type liquids, such as monohydric alcohols and secondary amides [32]. However, the materials examined in the present study are not classified as Debye-type liquids, although they are classified as alcohols. Sugar alcohols and trihydric alcohols are polyhydric alcohols and not monohydric alcohols. As can be recognized from the values of $\beta_{\mathrm{KWw}}$ listed in Table I, these materials exhibit significant non-Debye spectral shape at $\sim T_{g}$. Furthermore, all values of $m$ satisfy the relation between $m$ vs $\beta_{\mathrm{KWW}}$ proposed in Ref. [4] $\left(220-320 \beta_{\mathrm{KWW}}<m<280-320 \beta_{\mathrm{KWW}}\right)$.

In Fig. 5, the $\mathrm{OH}$ group orientational correlation factors for various polyhydric alcohols are plotted against temperature. It can be seen that all loci are very close and that the values of $g$ increase with decreasing temperature. From these results, we suggest that the probability for hydrogen-bond formation is approximately identical for these polyhydric alcohols, and that hydrogen-bond formation becomes more probable with decreasing temperature.

\section{CONCLUSIONS}

In our investigation, we have derived two results from the dynamic and static quantities obtained by BDS. From the former, we found that the temperature dependence of the relaxation time is strongly dependent on the number of $\mathrm{OH}$ groups per molecule, while molecular size has only a weak effect. From the latter, we suggest that the response of hydrogen bonding to decreasing temperature is similar among a variety of polyhydric alcohols. Based on these two experimental results, we speculate that the relaxation dynamics of supercooled polyhydric alcohols can be described by a network composed of nodes having $N_{\mathrm{OH}}$ connecting sites and bonds with a temperature-dependent connection probability.

\section{ACKNOWLEDGMENTS}

This work was partly supported by KAKENHI (Grant No. 19340116, MEXT of Japan) and Clark Memorial Foundation.
[1] E. Donth, The Glass Transition: Relaxation Dynamics in Liquids and Disordered Materials (Springer, Berlin, Heidelberg, New York, 2001).

[2] K. Binder and W. Kob, Glassy Materials and Disordered Solids (World Scientific, Singapore, 2005).
[3] C. A. Angell, J. Non-Cryst. Solids 131-133, 13 (1991).

[4] R. Böhmer, K. L. Ngai, C. A. Angell, and D. J. Plazek, J. Chem. Phys. 99, 4201 (1993).

[5] K. Kunal, C. G. Robertson, S. Pawlus, S. F. Hahn, and A. P. Sokolov, Macromolecules 41, 7232 (2008). 
[6] C. León, K. L. Ngai, and C. M. Roland, J. Chem. Phys. 110 11585 (1999).

[7] A. Döß, M. Paluch, H. Sillescu, and G. Hinze, Phys. Rev. Lett. 88, 095701 (2002); J. Chem. Phys. 117, 6582 (2002).

[8] K. L. Ngai and M. Paluch, J. Phys. Chem. B 107, 6865 (2003).

[9] A. Minoguchi, K. Kitai, and R. Nozaki, Phys. Rev. E 68, 031501 (2003); A. Minoguchi, T. Kaneko, H. Sotokawa, and R. Nozaki, J. Non-Cryst. Solids 352, 4742 (2006).

[10] H. Cohen and D. Turnbull, J. Chem. Phys. 31, 1164 (1959); D. Turnbull and H. Cohen, ibid. 34, 120 (1961).

[11] M. Nakanishi and R. Nozaki, Phys. Rev. E 81, 041501 (2010).

[12] Trihydric alcohols with longer chains $\left(N_{\mathrm{C}}=8-10\right)$ were also examined, but these crystallize too easily to make BDS measurements.

[13] M. Nakanishi, Y. Sasaki, and R. Nozaki, Rev. Sci. Instrum. 81, 123902 (2010).

[14] Although threitol and heptanetriol are also solid at room temperature, the extra measurements were not carried out on them in the present study because of the high price of these materials.

[15] U. Schneider, P. Lunkenheimer, R. Brand, and A. Loidl, J. NonCryst. Solids 235-237, 173 (1998).

[16] S. Havriliak and S. Negami, Polymer 8, 161 (1967).

[17] K. S. Cole and R. H. Cole, J. Chem. Phys. 9, 341 (1941).

[18] R. Kohlrausch, Pogg. Ann. Phys. 12, 393 (1847); G. Williams and D. C. Watts, Trans. Faraday Soc. 66, 80 (1970).
[19] F. Alvarez, A. Alegría, and J. Colmenero, Phys. Rev. B 44, 7306 (1991).

[20] C. M. Roland, R. Casalini, R. Bergman, and J. Mattsson, Phys. Rev. B 77, 012201 (2008).

[21] R. Chelli, P. Procacci, G. Cardini, R. G. D. Valle, and S. Califano, Phys. Chem. Chem. Phys. 1, 871 (1999); R. Chelli, P. Procacci, G. Cardini, and S. Califano, ibid. 1, 879 (1999).

[22] S. Sudo, N. Shinyashiki, and S. Yagihara, J. Mol. Liq. 90, 113 (2001).

[23] T. Psurek, S. Maslanka, M. Paluch, R. Nozaki, and K. L. Ngai, Phys. Rev. E 70, 011503 (2004).

[24] R. Nozaki, H. Zenitani, A. Minoguchi, and K. Kitai, J. NonCryst. Solids, 307-310, 349 (2002).

[25] M. Tatsumisago, B. L. Halfpap, J. L. Green, S. M. Lindsay, and C. A. Angell, Phys. Rev. Lett. 64, 1549 (1990).

[26] R. Böhmer and C. A. Angell, Phys. Rev. B, 45, 10091 (1992).

[27] J. G. Kirkwood, J. Chem. Phys. 7, 911 (1939).

[28] H. Fröhlich, Theory of Dielectrics (Oxford University Press, London, 1958).

[29] Handbook of Chemistry: Pure Chemistry, 5th ed., edited by Y. Iwasawa et al. (Maruzen, Tokyo, 2004).

[30] CRC Handbook of Chemistry and Physics, 86th ed., edited by D. R. Lide (CRC/Taylor and Francis, Boca Raton, FL, 2004).

[31] Material Safety Data Sheet for 3-Methyl-1,3,5-pentanetriol (Sigma-Aldrich, St. Louis, MO, 2006).

[32] L. Wang and R. Richert, J. Chem. Phys. 123, 054516 (2005). 